\section{Welche Bedeutung hat die Unterscheidung zwischen offener und geschlossener Lungentuberkulose für die Bekämpfung der Tuberkulose?}

Von Dr. E. Hartmann, Facharzt für Lungenkrankheiten in Magdeburg.

Bei der besonderen Wichtigkeit, die die Bekämpfung der Tuber. kulose in jetziger Zeit hat, sei es mir gestattet, zu einer Frage kurz Stellung zu nehmen, die von mehr als wissenschaftlichem Interesse ist, nämlich ob die allgemein verbreitete strenge Unterscheidung der Lungentuberkulose in "offene" und , geschlossene" Berechtigung und praktischen Wert hat.

Mit großer Beharrlichkeit hat sich diese Bezeichnung bis heute gehalten und wird in den Gutachten und Statistiken der Heilstätten Versicherungsanstalten, Fürsorgestellen, Militärbehörden usw. beson. ders angeführt. Sie ist oft von bestimmendem Einfluß für die $\mathrm{Be}$, urteilung des Falles und die zu ergreifenden Maßnahmen. Namentlich für die Frage der militärischen Verwendbarkeit war sie von einschneidender Bedeutung.

Zumeist verstand oder versteht man unter „offener" Lungen. tuberkulose solche mit Bazillen im Auswurf, unter "geschlossener" Lungentuberkulose solche ohne Bazillen im Auswurf. Andere machen den Unterschied entsprechend dem Vorhandensein oder Fehlen von Auswurf.

Wenn man sich überlegt, daß jede aktive Lungentuberkulose, wie u. a. die Untersuchungen von Richet ${ }^{1}$ ) ergeben haben, von Anfang an anatomisch als eine offene anzusehen ist, so ist in klinischem Sinne eine Unterscheidung der Lungentuberkulose in eine offene und geschlossene Form hinfällig, und es hat streng genommen jeder an aktiver Lungentuberkulose Erkrankte als ansteckend zu gelten.

Ferner ist das Ergebnis der Sputumuntersuchung un. $z$ uverlässig, weil von vielen Zufälligkeiten abhängig, und der negative Ausfall keineswegs entscheidend. Auf die Frage nach Auswurf wird sehr häufig von Patienten eine verneinende Ant. wort gegeben, womit sich der Arzt leider oft begnügt. Entweder die Kranken wissen nicht, daß sie Auswurf haben, da sie ihn ver. schlucken, wie dies die meisten Kinder tun, oder "so ein bißchen Schleim", der beim Räuspern morgens herauskommt, wird von ihnen nicht gerechnet. Dabei kann man es fast täglich erleben, daß der Patient, der angeblich keinen Auswurf hat, das trotzdem eingehändigte Untersuchungsglas prompt mit Auswurf wiederbringt, in dem dann oft zahlreiche Tuberkelbazillen nachzuweisen sind.

Die schwere Unterlassungssünde, sich mit einer einmaligen negativ ausgefallenen Sputumuntersuchung zu begnügen, dürfte eigent. lich heute nicht melır vorkonnmen, und doch trifft man sie noch täg lich an. Manchmal werden erst im zehnten oder gar noch spăteren Präparate Bazillen gefunden. Ist es doch eine bekannte Tatsache, da $B$ viele Lungentuberkulöse nicht fortwährend Bazillen ausscheiden bzw. Auswurf produzieren, sondern nur zeitweise, in kleineren oder größeren Zwischenräumen; namentlich bei alten Phthisikern trifft man diese Erscheinung gar nicht selten an. Dazu kommt noch, daB häufig ungeeignetes Sputum (Sekret aus dem Nasenrachenraum) untersucht wird oder es sonst an der nötigen Gründlichkeit der Untersuchung mangelt, indem diese nicht inmer von wissenschaft. lich und technisch auf der Höhe stehenden Laboratorien ausgeführt wird, die bewährten Anreicherungsverfahren nicht herangezogen werden u. dgl.

Selbst wenn auch in einem Sputumkeine Tuberkel. bazillen gefunden wurden, so ist dieses doch nicht

1) Presse,méd. 1917 Nr. 49 (Ref. M.'m. W. 1919). 
ohne weiteres als nicht infektiös zu betrachten, wie die Verimpfungen solchen Sputums am Versuchstier gezeigt haben. (Curschmann.)

Es ist daher nach allen diesen Tatsachen nicht verständlich,

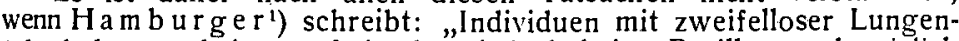
tuberkulose und Auswurf, in dem jedoch keine Bazillen nachweislich sind, sind nicht ansteckend, wenigstens so lange nicht, als eben keine Tuberkelbazillen nachweislich sind."

Außerdem - auch wenn kein Auswurf zu erlangen ist, bleibt immer noch die Möglichkeit der Tröpfcheninfektion durch die beim Husten (oder Niesen, Lachen) aus der Tiefe herausgeschleuderten Tuberkelbazillen. Leider wird dieser Art der Ansteckung heute im allgemeinen viel zu wenig Beachtung geschenkt und die Bedeutung der Sputuminfektion einseitig in den Vordergrund gedrängt.

Unsere heutige Zeit leidet an einer Ueberschätzung der bakteriologischen Untersuchungsbefunde. Wir sehen das ja auch bei anderen Krankheitszuständen. Bei der Tuberkulose bringt dieser Fehler die große Gefahr mit sich, daß sich bei negativem bakteriologischen Ergebuis der Patient und leider auch der Arzt zum schwersten Schaden des Kranken und eventuell dessen Ungebung oft über die ernste Natur der Krankheit täuscht.

Die Einteilung in offene und geschlossene Tuberkulose nach dem Bazillennachweis itn Auswurf hat aber, abgesehen von allen anderen Folgen, weittragende praktische Bedeutung für die gesamte Fürsorgetätigkeit:

Es ist die Rede davon, daB für „offene“ (in obigem Sinne) Tuberkulose die Anzeige p flicht gesetzlich eingeführt werden soll. Nach dem Gesagten liegt es auf der Hand, daß eine große - - wenn nicht sogar die größere - Anzahl ansteckungsfähiger Tuberkulöser dabei nicht erfaßt würde. Der Erfolg der Maßnahme wird dadurch schon nahezu illusorisch. Ferner ist zu bedenken, daß sehr viele, wahrscheinlich sogar die meisten Patienten, um sich der ihnen unbequemen Maßnahmen, die die Feststellung einer "offenen" Tuberkulose für sie im Gefolge haben würde, zu entziehen, ihren Auswurf einfach verheimlichen würden. Da der Arzt das Untersuchungsmaterial hier nicht wie bei anderen Organen eigenhändig entnehmen kann, so müßte wenigstens, um einigermaßen exakt vorzugehen, die Entleerung des Auswurfes in seinem Beisein geschehen (und das nicht nur einmil, sondern bei negativem bakteriologischen Befunde häufig!) $\mathrm{Daß}$ dies bei der ungelieuren Verbreitung der Tuberkulose unmöglich ist, ist klar, ebenso wie es ausgeschlossen und erfolglos sein würde, alle auf ihren Auswurf zu Untersuchenden eine Zeitlang in einem Krankenhause oder einer anderen Untersuchungsstation zu internieren. Man ist eben lediglich anf den Patienten selbst angewiesen.

Die bisherige Klassifizierung in offene und ge schlossene Lungentuberkulose is nach vorstehenden Ausführungen weder klinisch berechtigt noch in praktischer Hiusicht wertvoll, ja sogar unter Umständen schädlich. Ich habe daher diese Bezeichnung schon seit langem aufgegeben und mit mir gewiß wohl eine Anzahl anderer Fachkollegen. Wenn man vom Standpunkte der $F$ ürsorgetätigkeit eine Unterscheidung haben will, so wähle mian lieber die Ausdrücke ,ansteckungsfähige" und "nicht ansteckungsfähige" Tuberkulose, wobei diese Begriffe bei dem wechselvollen Verlaufe der Lungentuberkulose nur "zeitlich" zu verstehen sind. Die Unterscheidung ist nicht einseitig nach dem Sputumbefund zu machen, sondern nach folgenden Gesichtspunkten:

Unter die erste Rubrik wäre zu rechnen: allgemein gesprochen jede aktive Lunge ntuberkulose, kentitlich in erster Linie an dem Vorhandensein feuchter Rasselgeräusche. Wo feuchte Rasselgeräusche sind, da ist auch Sekretbildung vorhanden, dieses Sekret muß als infektiös angesehen werden, ob es nun als Auswurf oder in Tröpfchenform an die Außenwelt gelangt. Feuchte Rasselgeräusche als Zeichen von Sekretbildung - - wenn auch noch so spärlich und schwierig hörbar - habe ich auch in beginnenden Fällen so gut wie nie vermißt. Ist Lungenauswurf vorhanden (ob mit oder ohne Bazillen), so ist der Kranke eo ipso als ansteckungsfähig zu betrachten, doch habe ich noch nie erlebt, daß dann feuchte Rasselgeräusche fehlen, es sei denn, daB der Patient kurz vorher expektoriert hat was sich aber bei wiederholter Untersuchung feststellen läßt. Die Diagnose aktive Lungentuberkulose wird weiterhin durch den sonstigen klinischen und röntgenologischen Befund gesichert. Dabei soll nicht übersehen werden, daß der Röntgenbefund noch negativ sein kann, während physikalisch bereits deutliche Veränderungen bestehen.

Hier ausgenommen und zul Rubrik II zu rechnen sind die aktiven Fälle die im allerersten Beoinn sind und noch keine eindeutigen physikalischen bzw. röntgenologischen Veränderungen an den Lungen anfweisen (primäres Latenzstadium nach $\mathrm{Asch}$ of $\mathrm{f}-\mathrm{Nicol}$ ), z. B. die leichten Hilus-Bronchialdrüsenveränderungen, wo es noch nicht zu einem "Katarrh" gekommen ist. Die Diagnose wird nur selten gestellt, weil eindeutige physikalisch nachweisbare Veränderungen fehlen und die meisten Kranken mangels gröBerer Beschwerden den Arzt noch nicht aufsuchen. Hierher gehört auch die unter dem Bilde einer Pleuritis (sonst ohne nachweisbare Lungenerscheinungen) auftretende Lungentuberkulose.

Die Fälle der zweiten Rubrik werden ferner dargestellt durch alle, die nach klinischem Befunde und genauer Beobachtung "inaktiv“ oder genauer gesagt, symptomlos geworden sind, insbesondere keinen Lungenauswurf und keinen Husten mehr haben. Feuchte Rasselgeräusche wird man dann ebenfalls vermissen, z. B. können durch künstlichen Pueumotiorax symptomlos Gewordene dazu rechnen.

Wer klinisch ,ausgeheilt" ist (mehrere Jahre völlig symptomlos und voll arbeitsfähig), kann nicht mehr als krank bezeichnet werden und fällt daher nicht unter diese Rubrik.

Dieses wären meines Erachtens Richtlinien, nach denen praktisch gearbeitet werden kann. Deshalb sollte der Arzt jedem Patienten, bei dem er eine aktive Lungentuberkulose feststellt, die nötigen Verhaltungsmaßregeln zum Schutze seiner Umgebung geben.

Wenn eine Anzeigepflicht für ansteckungsfähige Tuberkulose eingeführt werden soll, so müßte sich diese auf alle an aktiver Lungentuberkulose Erkrankte erstrecken. So bestechend der Gedanke einer Erfassung aller ansteckungsfähigen Tuberkulösen zur Eindämmung der Weiterverbreitung der Krankheit ist, so könnte eine solche Anzeigepflicht nur einen statistischen Wert haben; denn eine behördliche wirklich erfolgreiche Fürsorge in diesem Umfange muß bei der ungeheueren Verbreitung der Tuberkulose - namentlich in einem besiegten Deutschland - leider versagen. 